\title{
Effect of 6-Mercaptopurine on Rat Placenta
}

\author{
Satoshi FURUKAWA ${ }^{1) *}$, Koji USUDA $^{1)}$, Masayoshi ABE $^{1)}$, Seigo HAYASHI ${ }^{1)}$ and Izumi OGAWA ${ }^{1)}$ \\ ${ }^{1)}$ Biological Research Laboratories, Nissan Chemical Industries, Ltd., 1470 Shiraoka, Minamisaitama, Saitama 349-0294, Japan
}

(Received 13 November 2007/Accepted 1 February 2008)

ABSTRACT. In order to investigate the toxic effects of 6-mercaptopurine (6-MP) on placental development, we examined sequential morphology in the placentas from rats exposed to 6-MP. 6-MP was intraperitoneally administered at $60 \mathrm{mg} / \mathrm{kg}$ during gestation days (GDs) 11 to 12 , and the placentas were sampled on GD 13, 15 or 21. In the 6-MP-treated group, maternal body weight suppression, increased death embryo/fetus ratio and some malformations were observed. The placenta weights were decreased on GDs 15 and 21 . Macroscopically, placentas on GD 21 were small, brittle and thin with a white peripheral rim. Histopathologically, in the labyrinth zone, 6-MP treatment mainly evoked decreased mitosis on GDs 13 and 15, increased apoptotic cell on GDs 13, 15 and 21 and thinning on GDs 15 and 21. In the basal zone, 6-MP evoked decreased mitosis on GDs 13, and PAS-positive material in the spongiotrophoblasts was still detected on GD 15. Thickening of the basal zone was observed with cytolysis of glycogen cells, apoptosis and an increased number of composed cells on GD 21. In conclusion, 6-MP administration in pregnant rats induced growth arrest of the labyrinth zone and developmental delay in the basal zone, leading to small placentas. The fetotoxicity of 6-MP may be responsible for its direct anti-proliferative effects and resulting placental dysfunction.

KEY WORDS: 6-mercaptopurine, apoptosis, placenta, rat.

J. Vet. Med. Sci. 70(6): 551-556, 2008

The placenta is a multifaceted organ that performs a number of essential and highly differentiated functions which are modified throughout gestation. The placenta secures the embryo and fetus to the endometrium and releases a variety of steroid and protein hormones which convert the physiology of a female to that of a pregnant female. It is also responsible for maternoembryonic exchange of $\mathrm{O}_{2} / \mathrm{CO}_{2}$ and nutrient/metabolite requirements during embryonic development, as well as a protective barrier against xenobiotics. Placental growth and function affect biological significance in the pregnant maintenance and prenatal life. Therefore, chemical-induced alteration or deviation of placental function in the maternal and extraembryonic tissue can ultimately lead to pregnancy loss, congenital malformation and fetal death.

6-mercaptopurine (6-MP) has been used as an anti-leukemic drug, and is also a metabolite of azathioprine, an immunosuppressive drug. 6-MP induces embryo-toxic effects, including a reduction in the number of implantation sites and is also known as a teratogen, inducing limb defects, micrognathia, ventral hernia, skeletal, urogenital, central nervous system and ocular anomalies, cleft palate, diaphragmatic hernia, etc. in rats, mice and rabbits $[1,2,13,18,19]$. On the other hand, Bargnoier et al. suggested that placental tissues may be more sensitive to 6-MP than the fetus, and it is known that the placentas from 6-MP-treated rats show a disproportionate reduction in thickness of the labyrinth zone with fibrinous degeneration of trophoblasts [3, 24]. However, there are few reports about the sequential morphologi-

\footnotetext{
* Correspondence to: Furukawa, S., Toxicology Group, Toxicology \& Environmental Science Department Biological Research Laboratories, Nissan Chemical Industries, Ltd., 1470 Shiraoka, Minamisaitama, Saitama 349-0294, Japan.

e-mail: furukawa@nissanchem.co.jp
}

cal details of these changes in the 6-MP-exposed placenta [9]. In order to investigate the toxic effects of 6-MP on placental development, we examined sequential morphology in placentas from rats exposed to $6-\mathrm{MP}$ at $60 \mathrm{mg} / \mathrm{kg}$ during gestation days (GDs) 11 to 12 .

\section{MATERIALS AND METHODS}

Animals: A total of 24 pregnant specific pathogen-free Crlj:CD(SD) rats (Charles River Laboratories Japan, Inc., Japan), at approximately 10-14 weeks of age, were purchased. The animals were single-housed in wire-mesh cages in an air-conditioned room $\left(22 \pm 2{ }^{\circ} \mathrm{C}\right.$; humidity, $55 \pm$ $10 \%$; light cycle, $12 \mathrm{hr} /$ day). Feed (Oriental Yeast Co., Ltd., Japan) and water were available ad libitum.

Experimental design: GD 0 was designated as the day when the presence of a vaginal plug was identified. 6-MP (Aldrich, U.S.A.) was suspended in olive oil and administered intraperitoneally (i.p.) $(0.5 \mathrm{ml} / 100 \mathrm{~g}$ body weight $)$. The pregnant rats were randomly allocated to 2 groups of 12 rats each. Animals were treated daily at dose levels of 0 (olive oil only) or $60 \mathrm{mg} / \mathrm{kg}$ during GDs 11 to 12 . The dose level of $60 \mathrm{mg} / \mathrm{kg}$ i.p. on GD 11 or 12 was previously reported to have an effect on placentas in rats [3, 24]. All treatments were given between 10 and 11 a.m. Maternal body weight was recorded on GDs $0,11,12,13,15$, and 21 . Four rats from each group were sampled on GD 13, 15, or 21. Dams were euthanized by exsanguination under diethyl ether anesthesia and necropsied. All embryos/fetuses and half of the placentas were removed from the uterus and weighed. This experiment was conducted according to the Guidelines for Animal Experimentation, Japanese Association for Laboratory Animal Science, 1987.

Histopathological examination: The placentas, which 
were not removed from the uterus, were fixed in $10 \%$ neutral buffered formalin. These tissues were embedded in paraffin, sectioned at a $4-\mu \mathrm{m}$ thickness, and stained routinely with hematoxylin and eosin (H\&E) and periodic acid Schiff (PAS) for histopathological examination. Immunohistochemical staining, in situ TdT-mediated dUTP nick end labeling (TUNEL) (ApopTag ${ }^{\circledR}$ : Chemicon International, U.S.A.) was performed on these tissues. Thickness of the labyrinth and basal zone close to the central portion were measured in two or five randomly chosen placentas from each dam with the aid of an image analyzer (IPAP; Processor for Analytical Pathology, Sumika Technoservice Co. Japan). The numbers of mitotic cells and apoptotic cells (except giant cells) in the labyrinth and basal zone on GDs 13,15 and 21 were counted in five different fields $(\times 400)$ for each placenta.

Statistical analysis: Means and standard error (SE) of the individual litter values were calculated. Continuous data were analyzed with the F test. When variances were homogeneous, the Student $t$-test was performed. The AspinWelch $t$-test was performed when variances were not homogeneous. The Wilcoxon rank sum test was employed for nonparametric data, such as the dead embryo/fetus ratio. The level of significance was set at $P<0.05,0.01$ and 0.001 .

\section{RESULTS}

All 6-MP-treated dams clinically were debilitated for two days after the second treatment. When compared with the control group, a significant reduction in bodyweight gain of dams in the treated group was seen during GDs 12 to 21 (Fig. 1). On GD 21, maternal body weight suppression in the treated group was $74 \%$ of that of the control group.

Table 1 shows the total numbers and weights of the live embryos/fetuses, the dead embryo/fetus ratio and the placenta weights at each sampling time point. The dead embryo/fetus ratio in the treated group on GD 21 was significantly higher than that of the control group. The embryo/ fetus weights in the treated group on GDs 13, 15 and 21 were significantly lower than those of the control group and were reduced by $23 \%, 63 \%$ and $79 \%$, respectively. The fetuses in the treated group on GD 21 were small with micrognathia, umbilical hernia/omphalocele, anal atresia, phocomelia, digit anomalies, tail defects, and ectopic pinna (Fig. 2a). The placenta weights in the treated group on GDs 15 and 21 were significantly lower than those of the control group and were reduced by $47 \%$ and $55 \%$, respectively. Macroscopically, the placentas on GD 21 were small, brittle and thin with a white peripheral rim, which appeared to be wider in comparison with the control group (Fig. 2b).

Histopathologically, on GDs 13 and 15, an increase in apoptotic cells, which were characterized by pyknosis or karyorrhexis, phagocytosis and cell debris on H\&E sections (Fig. 2c), and positively stained by the TUNEL method (Fig. $2 \mathrm{~d}$ ), and a decrease in mitotic trophoblasts were seen in the labyrinth zone in the treated group. There were decreased trophoblasts, a reduction in thickness of the trophoblastic septa and irregular dilatation of maternal sinusoids with deposition of fibrin in the treated group (Fig. 2e). In the basal zone, there was decreased mitotic spongiotrophoblasts

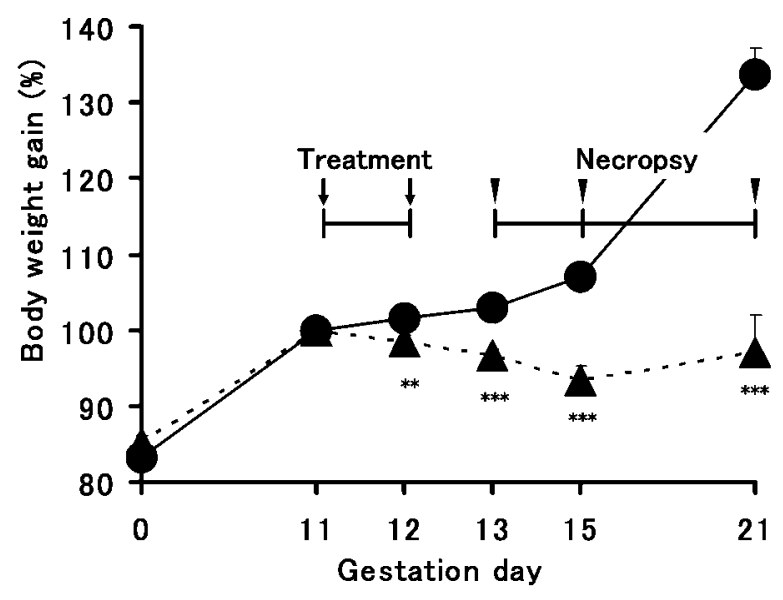

Fig. 1. Maternal body weight gain. $\mathbf{\Delta}$ : Control, 0 : 6-MP. Each value represents mean $\pm \mathrm{SE}$. $* * * * *$ : Significantly different from control at $P<0.01,0.001$ (Student $t$-test).

Table 1. Effect of 6-mercaptopurine on embryos/fetuses and placentas

\begin{tabular}{|c|c|c|c|c|c|}
\hline $\begin{array}{c}\text { Gestation } \\
\text { day }\end{array}$ & Group & $\begin{array}{l}\text { Total No. of } \\
\text { live embryos/ } \\
\text { fetuses }\end{array}$ & $\begin{array}{c}\text { Dead } \\
\text { embryo/fetus } \\
\text { ratio }(\%)^{\text {a) }}\end{array}$ & $\begin{array}{c}\text { Embryo/fetus } \\
\text { weight } \\
(\mathrm{g})^{\mathrm{a})}\end{array}$ & $\begin{array}{l}\text { Placenta } \\
\text { weight } \\
(\mathrm{g})^{\mathrm{a})}\end{array}$ \\
\hline \multirow[t]{2}{*}{13} & Control & 53 & $3.6 \pm 3.6$ & $0.072 \pm 0.002$ & $0.126 \pm 0.019$ \\
\hline & 6-mercaptopurine & 54 & $6.8 \pm 2.7$ & $0.055 \pm 0.005 *$ & $0.114 \pm 0.001$ \\
\hline \multirow[t]{2}{*}{15} & Control & 57 & $1.9 \pm 1.9$ & $0.288 \pm 0.012$ & $0.265 \pm .025$ \\
\hline & 6-mercaptopurine & 55 & $12.5 \pm 12.5$ & $0.106 \pm 0.018^{* * *}$ & $0.139 \pm 0.002^{\$}$ \\
\hline \multirow[t]{2}{*}{21} & Control & 60 & $0.0 \pm 0.0$ & $5.235 \pm 0.197$ & $0.432 \pm 0.013$ \\
\hline & 6-mercaptopurine & 31 & $38.8 \pm 14.7^{\#}$ & $1.086 \pm 0.154 * * *$ & $0.196 \pm 0.033 * * *$ \\
\hline
\end{tabular}

Mean \pm SE.

a): Mean of individual litter values.

$*, * * *$ : Significantly different from control at $P<0.05,<0.001$, respectively (Student $t$-test).

\$: Significantly different from control at $P<0.05$ (Aspin-Welch $t$-test).

\#: Significantly different from control at $P<0.05$ (Wilcoxon rank sum test). 
on GD 13. Some spongiotrophoblasts around multiple clusters of glycogen cells contained PAS-positive material until GD 15 in the treated group (Fig. 2f), although spongiotrophoblasts contained little or no PAS-positive material on GD 15 in the control group. The clusters of glycogen cells consisted of small and irregular-shaped cells as compared with the controls. On GD 21, the above-mentioned lesions in the labyrinth zone became marked with degeneration and necrosis of trophoblasts in the treated group (Fig. $2 \mathrm{~g}$ ). In the basal zone, there was an increased number of composed cells (spongiotrophoblasts, glycogen cells and giant cells), presence of focal necrosis, calcium deposition, increased apoptosis and multiple hemorrhagic cysts, which consisted of cytolysis involving multiple clusters of residual glycogen cells (Fig. 2h). Some giant cells had large vesicular nuclei, which contained PAS-positive materials. At the edge of placenta, an increase in accumulation of cell debris, including a degenerated Reichert's membrane, was observed in comparison with the control group. There was no histopathological change in the decidua basalis during GDs 13 to 21 .

There was significant thinning of the labyrinth zone on GDs 15 and 21, and thickening of the basal zone on GD 21 in comparison with the control group (Fig. 3). No change in diameter of either the labyrinth or basal zones was noted on GD 13. There were significantly increased apoptotic cells on GDs 13, 15 and 21, and decreased mitotic cells on GDs 13 and 15 in the labyrinth zone, and decreased mitotic cells on GD 13 and increased apoptotic cells on GD 21 in the basal zone in the treated group (Fig. 4).

\section{DISCUSSION}

The results indicated that maternal intraperitoneal administration of 6-MP at $60 \mathrm{mg} / \mathrm{kg} /$ day during GDs 11 to 12 induced maternal body weight suppression, increased death embryo/fetus ratio, some malformations and small placentas. 6-MP treatment mainly evoked thinning of the labyrinth zone with increased apoptotic cells and decreased mitosis, and thickening of the basal zone with cytolysis of glycogen cells and an increased number of composed cells.

6-MP is a purine antimetabolite with high toxic sensitivity against rapidly dividing cells due to DNA damage [7, 8, 21], that promotes rapid apoptotic cell death [14]. It is known that 6-MP inhibits DNA and RNA synthesis in the fetus and placenta inducing their damage $[3,19]$. In early placental development, the ectoplacental cone differentiates into trophoblasts in the labyrinth zone and spongiotrophoblasts in the basal zone, and these cells exhibit high proliferative activity [20]. The volume of the whole placenta is increased linearly, caused by the increased contribution of the labyrinth zone to the placenta [6]. Therefore, it is suspected that 6-MP induces DNA damage in trophoblasts, leads to apoptosis and mitotic inhibition in the labyrinth zone and causes a lack of the cell populations required for later normal histogenesis, resulting in a small placenta. On the other hand, 6-MP induced mitotic inhibition of spongiotrophoblasts, but did not increase apoptosis in the basal zone on GDs 13 and 15. It seems that the susceptibility to 6-MP in the labyrinth and basal zones is completely different. Ethylnitrosourea (ENU) [17], 1- $\beta$-D-arabinofuranosylcytosine (Ara-C) [28] and busulfan [10] induce apoptosis and impair cell proliferation in trophoblasts in the labyrinth zone due to DNA damage. These compounds also induce less, or no, apoptosis in the spongiotrophoblasts in the basal zone, the same as 6-MP. Since it is known that the proliferative activity of spongiotrophoblasts diminishes earlier than that of trophoblasts with advancing pregnancy [16], the difference in susceptibility to these anticancer drugs between trophoblasts and spongiotrophoblasts may be due to the difference in the proliferative activity of each cell related to the developmental stage.

In normal placental development, the basal zone is composed of small cytotrophoblastic elements, which are diffusely PAS-positive materials as they contain glycogen on GD $12[5,6]$. Subsequently, the cytotrophoblastic elements undergo cellular differentiation into glycogen cells and spongiotrophoblasts, which have little or no PAS-positive material after GD 14. Glycogen cells exhibit pyknosis and cytolysis on GD 18, and almost disappear until GD 21. The basal zone size reduces with the placental developmental process after GD 15. In this study, PAS-positive material in the spongiotrophoblasts was still detected on GD 15 and it seemed that regression of the basal zone on GD 21 was later than normal developmental process. Thus, it is suggested that 6-MP induces delay in the developmental process in the basal zone. From the present study, we could not prove the mechanisms for developmental delay of the basal zone, although it may be related to the mitotic inhibition of spongiotrophoblasts.

The macroscopically white peripheral rim in the 6-MPtreated placenta consisted of cell debris and a degenerated Reichert's membrane. The same changes were observed in busulfan-induced small placentas [10]. We suspected that the growth arrest of the labyrinth zone induced the poor development of the decidua basalis, resulting in enlargement of the volume occupied by the decidua capularis as a compensatory reaction.

Maternal weight loss during pregnancy in rats induces a reduction in the placental and fetal weight, but is not necessarily associated with embryo lethality and teratogenicity [4]. No marked histopathological changes in trophoblasts are noted in the small placentas caused by maternal malnutrition, although placental glycogen stores are reduced [23]. Therefore, we speculated that 6-MP-induced fetal and placental abnormalities might not be due to systemic toxic maternal effects. On the other hand, it seemed that the direct anti-proliferative effect of 6-MP might be one of important factors in the fetal growth retardation and abnormalities. In addition, the placental size and structure along with developmental and pathological processes cooperate in placental transport and metabolic mechanisms to affect placental-fetal nutrient exchange. The trophoblasts in the labyrinth zone, in which the maternal and fetal blood come very close together, play pivotal roles in the maternal-fetal exchange, 

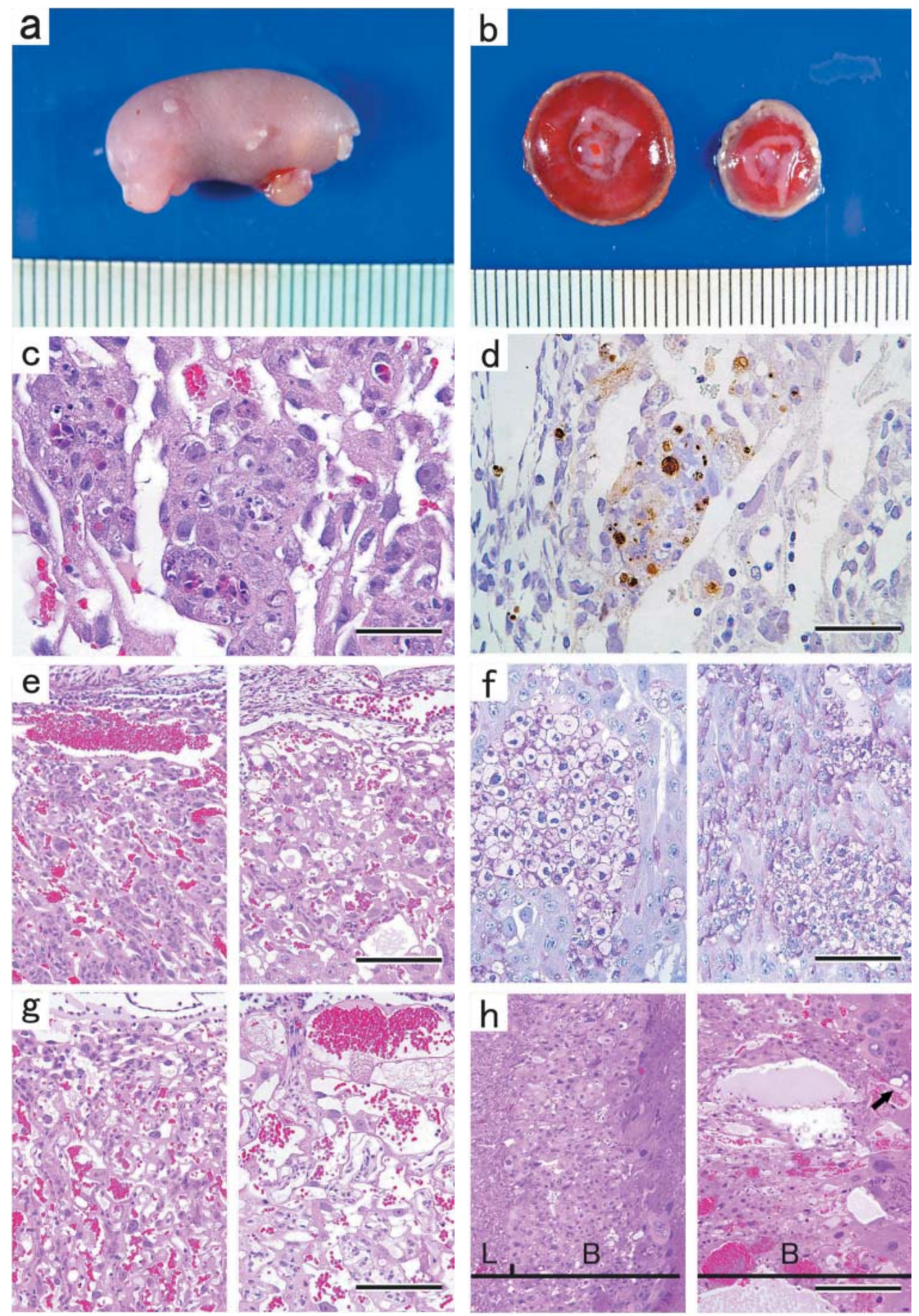

placental xenobiotic metabolism, placental endocrine function, and placental substance barrier [12, 25]. The basal zone is the site of production of protein, steroid and peptide hormones [6]. Thus, a small placenta results in reduced uterine blood flow to the placenta and transport to the fetuses, and is the major determinant of fetal growth retardation [11]. From the present study, we could not prove to what extent the placental dysfunction affects the fetal abnormalities and fetal growth retardation. It is known that some toxicants such as ENU [17], Ara-C [27], busulfan [10], T-2 


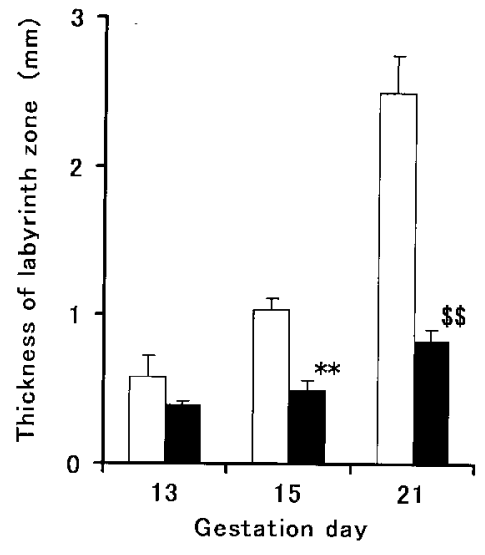

Labyrinth zone

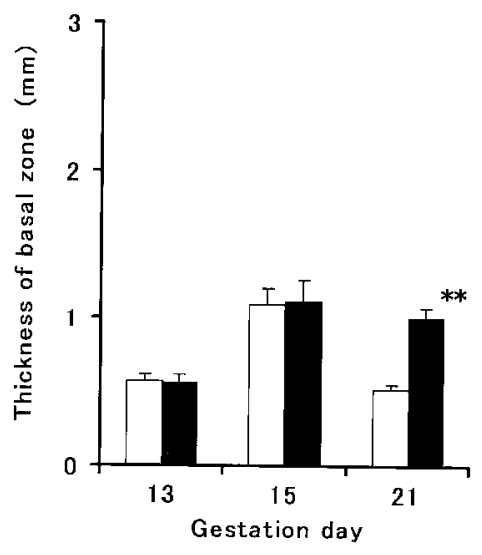

Basal zone

Fig. 3. Thickness of labyrinth and basal zones on GDs 13, 15 and 21. $\square$ : Control, $\square$ : 6MP. Each value represents mean \pm SE. **: Significantly different from control at $P<0.01$ (Student $t$-test). ${ }^{\$}$ : Significantly different from control at $P<0.01$ (AspinWelch $t$-test).

Mitotic cells

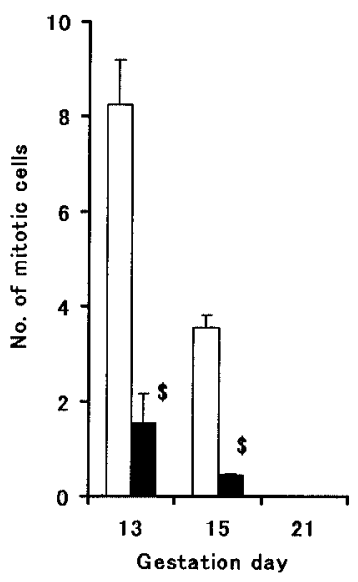

Apoptotic cells

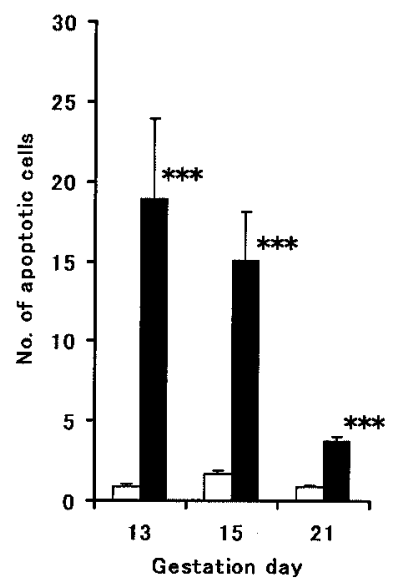

Mitotic cells

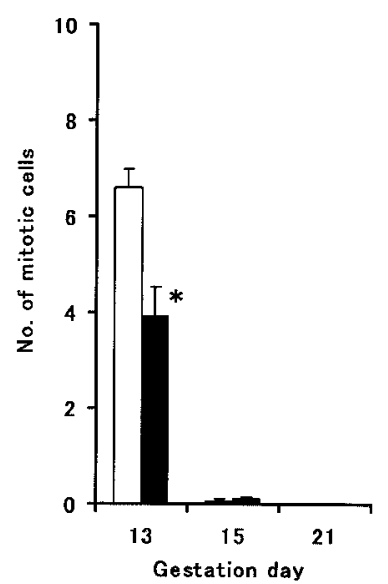

Apoptotic cells

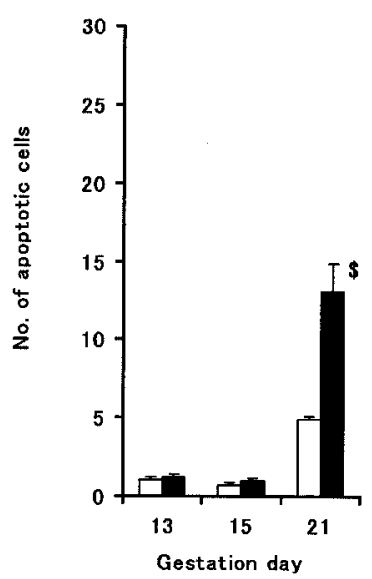

\section{Labyrinth zone}

\section{Basal zone}

Fig. 4. Mitotic and apoptotic cells in labyrinth and basal zones on GDs 13, 15 and 21. $\square$ : Control, $\square$ : 6-MP. Each value represents mean \pm SE. *, ***: Significantly different from control at $P<0.05,0.001$, respectively (Student $t$-test). ${ }^{\text {: }}$ : Significantly different from control at $P<0.05$ (Aspin-Welch $t$-test).

Fig. 2. Gross appearance of the fetus and placenta, and histopathological placenta findings. a: Gross appearance of a fetus in the 6-MP group on GD 21. Small, micrognathia, omphalocele, phocomelia, digit anomalies, tail defects, and ectopic pinna in a 6-MP-treated rat. b: Gross appearance in a control (left) and 6-MP (right) placenta on GD 21. Small placenta with a white peripheral rim, which appears wider in comparison with the control placenta. c: Labyrinth zone in 6-MP- treated-placenta on GD 13 (HE stain). Apoptotic cells, characterized by pyknosis or karyorrhexis, phagocytosis and cell debris, in trophoblastic septa. Bar $=75 \mu \mathrm{m}$. d: Labyrinth zone in 6-MP-treated-placenta on GD 13 (TUNEL stain). TUNEL-positive trophoblasts in trophoblastic septa. Bar=75 $\mu \mathrm{m}$. e: Labyrinth zone in control (left) and 6-MP (right) on GD 15 (HE stain). Decreased trophoblasts, a reduction in thickness of trophoblastic septa and irregular dilatation of maternal sinusoids in a 6MP-treated placenta. Bar $=300 \mu \mathrm{m}$. f: Basal zone in control (left) and 6-MP (right) on GD 15 (PAS stain). PAS-positive material in some spongiotrophoblasts around clusters of glycogen cells in 6-MP-treated placenta. Bar=150 $\mu \mathrm{m}$. g: Labyrinth zone in a control (left) and 6-MP (right) placenta on GD 21 (HE stain). Decreased number, degeneration of trophoblasts, a reduction in thickness of trophoblastic septa and irregular dilatation of maternal sinusoids with deposition of fibrin in a 6-MP-treated placenta. Bar=300 $\mu \mathrm{m}$. h: Basal zone in a control (left) and 6-MP (right) placenta on GD 21 (HE stain). Thickening of the basal zone with increased spongiotrophoblasts and giant cells, and multiple hemorrhagic cysts in a 6-MP-treated placenta. Large vesicular nuclei $(\mathbf{A})$ in giant cells. Bar=300 $\mu$ m. Labyrinth zone (L), Basal zone (B). 
toxin [22], azacytidine [15], adrenomedullin antagonist [26] etc., induce small placenta and fetal growth restriction in rats or mice, although their induced fetal abnormalities are different from the ones in 6-MP. Therefore, 6-MP-induced fetal abnormalities seem to be caused by its specific direct anti-proliferative effects during organogenesis. Meanwhile, the fetal intra-uterine growth retardation may not only result in the anti-proliferative effects of 6-MP, but also the disruption of placental functions during the fetal period.

In conclusion, 6-MP administration in pregnant rats induced growth arrest of the labyrinth zone and developmental delay in the basal zone, leading to small placentas. The fetotoxicity of 6-MP may be responsible for its direct anti-proliferative effects and resulting placental dysfunction.

ACKNOWLEDGEMENTS. The authors would like to thank Mr. Kiyoshi Kobayashi, Ms. Kaori Maejima, Ms. Hiromi Asako, Mr. Atsushi Funakoshi, Mr. Yoshinori Tanaka, Ms. Mayumi Seta, and Mr. Shigeru Iimura for their excellent technical assistance.

\section{REFERENCES}

1. Amemiya, K., Keen, C.L. and Hurley, L.S. 1986. 6-Mercaptopurine-induced alterations in mineral metabolism and teratogenesis in the rat. Teratology 34: 321-334.

2. Arakawa, T., Fujii, M. and Hayashi, T. 1967. Dilatation of cerebral ventricles of rat offsprings induced by 6-mercaptopurine administration to dams. Tohoku J. Exp. Med. 91: 143-148.

3. Bragonier, J.R. and Carver, M.J. 1968. The influence of 6-mercaptopurine on rat placenta and fetus. Biochem. Pharmacol. 17: 1689-1697.

4. Chahoud, I., Kuriyama, S.N. and Paumgartten, F.J. 2002. Maternal protein-and-energy restriction reduces the developmental toxicity of cyclophosphamide and hydroxyurea in rats. Toxicology 179: 137-149.

5. Cross, J.C. 2005. How to make a placenta: Mechanisms of trophoblast cell differentiation in mice-a review. Placenta $\mathbf{2 6}$ (Suppl. A): S3-S9.

6. Davies, J. and Glasser, S.R. 1968. Histological and fine structural observations on the placenta of the rat. Acta Anat. (Basel) 69: $542-608$.

7. Elion, G.B. 1967. Symposium on immunosuppressive drugs. Biochemistry and pharmacology of purine analogues. Fed. Proc. 26: 898-904.

8. Estlin, E.J. 2001. Continuing therapy for childhood acute lymphoblastic leukaemia: Clinical and cellular pharmacology of methotrexate, 6-mercaptopurine and 6-thioguanine. Cancer Treat. Rev. 27: 351-363.

9. Fein, A. and Gross, A. 1976. Effect of azathioprine on the implanting trophoblast in rats. Isr. J. Med. Sci. 12: 86-87.

10. Furukawa, S., Usuda, F., Abe, M., Hayashi, S. and Ogawa, I. 2007. Busulfan-induced apoptosis in rat placenta. Exp. Toxic. Pathol. 59: 97-103.

11. Garnica, A.D. and Chan, W.Y. 1996. The role of the placenta in fetal nutrition and growth. J. Am. Coll. Nutr. 15: 206-222.

12. Goodman, D.R., James, R.C. and Harbison, R.D. 1982. Placental toxicology. Fd. Chem. Toxic. 20: 123-128.
13. Hirsch, K.S. and Hurley, L.S. 1978. Relationship of dietary zinc to 6-mercaptopurine teratogenesis and DNA metabolism in the rat. Teratology 17: 303-313.

14. Hortelano, S. and Boscáî, L. 1997. 6-Mercaptopurine decreases the $\mathrm{Bcl}-2 / \mathrm{Bax}$ ratio and induces apoptosis in activated splenic B lymphocytes. Mol. Pharmacol. 51: 414-421.

15. Hossain, M.M., Nakayama, H. and Goto, N. 1995. Apoptosis in the central nervous system of developing mouse fetuses from 5-azacytidine-administered dams. Toxicol. Pathol. 23: $367-372$.

16. Iguchi, T., Tani, N., Sato, T., Fukatsu, N. and Ohta, Y. 1993. Developmental changes in mouse placental cells from several stages of pregnancy in vivo and in vitro. Biol. Reprod. 48: 188196.

17. Katayama, K., Ueno, M., Takai, H., Ejiri, N., Uetsuka, K., Nakayama, H. and Doi, K. 2002. Ethylnitrosourea induces apoptosis and growth arrest in the trophoblastic cells of rat placenta. Biol. Reprod. 67: 431-435.

18. Kury, G.S., Chaubo, S. and Murphy, L. 1968. Teratogenic effects of some purine analogues on fetal rats. Arch. Pathol. 86: $396-402$.

19. Matalon, S.T., Ornoy, A. and Lishner, M. 2004. Review of the potential effects of three commonly used antineoplastic and immunosuppressive drugs (cyclophosphamide, azathioprine, doxorubicin on the embryo and placenta). Reprod. Toxicol. 18: 219-230.

20. Peel, S. and Bulmer, D. 1977. Proliferation and differentiation of trophoblast in the establishment of the rat chorio-allantoic placenta. J. Anat. 124: 675-687.

21. Platzek, T., Schwabe, R., Rahm,U. and Bochert, G. 1994. DNA modification induced by 6-mercaptopurine riboside in murine embryos. Chem. Biol. Interact. 93: 59-71.

22. Rousseaux, C.G. and Schiefer, H.B. 1987. Maternal toxicity, embryolethality and abnormal fetal development in CD-1 mice following one oral dose of T-2 toxin. J. Appl. Toxicol. 7: 281288.

23. Rudge, M.V., Gomes, C.M., Calderon, Ide, M., Ramos, M.D., Abbade, J.F., de Oliveira, M.G. and da Silva, M.G. 1999. Study of the evolution of the placenta and fetal pancreas in the pathophysiology of growth retardation intrauterine due to restricted maternal diet. Sao Paulo Med. J. 117: 49-56.

24. Shrader, R.E., Hirsch, K.S., Levin, J. and Hurley, L.S. 1978. Attenuating effect of zinc on abnormal placental morphology in 6-mercaptopurine treated rats. Teratology 17: 315-325.

25 Takata, K., Fujikura, K. and Shin, B. 1997. Ultrastructure of the rodent placental labyrinth: A site of barrier and transport. $J$. Reprod. Dev. 43: 13-24.

26. Witlin, A.G., Li, Z.Y., Wimalawansa, S.J., Grady, J.J., Grafe, M.R. and Yallampalli, C. 2002. Placental and fetal growth and development in late rat gestation is dependent on adrenomedullin. Biol. Reprod. 67: 1025-1031.

27. Yamauchi, H., Katayama, K., Yasoshima, A., Uetsuka, K., Nakayama, H. and Doi, K. 2003. 1- $\beta$-D-Arabinofuranosylcytosine (Ara-C)-induced apoptosis in the rat fetal tissues and placenta. J. Toxicol. Pathol. 16: 223-229.

28. Yamauchi, H., Katayama, K., Ueno, M., Uetsuka, K., Nakayama, H. and Doi, K. 2004. Involvement of p53 in 1- $\beta$-Darabinofuranosylcytosine-induced trophoblastic cell apoptosis and impaired proliferation in rat placenta. Biol. Reprod. 70: 1762-1767. 